\title{
Radio Workshop Report: Public Awareness of Radio Interference
}

\author{
David G. Finley ${ }^{1}$ \\ National Radio Astronomy Observatory, P.O. Box O, Socorro, New \\ Mexico 87801 USA
}

This, the final radio workshop session, covered the vital topic of public outreach. A number of valuable ideas emerged, with at least one specific goal to be achieved in a relatively short amount of time.

First, an important point that perhaps should be expected to come out of such an international gathering is that there is no single solution or "one size fits all" outline for educational efforts aimed at the public and at policy-makers. There are differences in how the news media operate in different countries and cultures that must be taken into account. Also, regulatory agencies in different countries vary, sometimes dramatically, in their receptiveness to public input on policy and technical matters. In each country, therefore, the educational efforts must be tailored to the audiences and political structures and traditions prevailing there.

One audience that should prove receptive to our message is the "general" scientist and engineer - not an astronomer, but someone capable of readily grasping both the technical aspects of radio frequency interference and also the importance of the issue to radio astronomy.

As part of the effort to educate both the general public and the general scientist and engineer, we need to publicize radio astronomy research and the effects of radio interference on that research. Publicity materials such as press releases, brochures, videos, Web sites, etc., about research results from radio astronomy should mention the frequency at which the observation was made. If we show the public a beautiful image or significant new scientific discovery made with a radio telescope, the public should understand that producing it required use of radio spectrum.

In a number of countries there are annual events such as "Outreach Week" or "Astronomy Day," in which the research community is encouraged to present science to the public. Radio observatories and individual radio astronomers should participate in such events, raising the level of public awareness. These events often receive considerable publicity and we can use these opportunities to make the case for interference mitigation.

Many radio observatories have well-attended visitor centres. The audiences at these visitor centres already are mostly well disposed towards radio astronomy. We could make our point about radio interference to these audiences by making our visitor centres as RFI-compliant as possible. Having visitors enter a Faraday cage for example and explaining to them why it is necessary to prevent the

\footnotetext{
${ }^{1}$ The National Radio Astronomy Observatory is a facility of the National Science Foundation, operated under cooperative agreement by Associated Universities, Inc.
} 
microprocessors in the exhibits from interfering with the radio telescopes outside, could quickly and dramatically sensitize them to the interference issue.

Finally, it was pointed out that, despite the importance of the World Wide Web as a tool for education and communication, there does not exist, to our knowledge, any material on the Web specifically designed to explain the interference issue to the general public. Individual radio observatories have oftenextensive Web sites aimed at providing observers with information on their local RFI environments, but there are no "non-technical" Web sites on the topic.

To remedy this situation, we decided that NRAO will take the lead in producing a Web page or pages explaining the interference issue in terms understandable to the general public. This material will be either duplicated or linked to by any other observatory, by the IDA, the IAU and other astronomical institutions. In addition, we will seek to produce a Web repository of material and graphics that can be used for viewgraphs, providing a resource for anyone who wishes to talk about the interference issue in public lectures. 